\title{
In silico Prediction of Novel SARS-CoV 3CL pro Inhibitors: a Combination of 3D-QSAR, Molecular Docking, ADMET Prediction, and Molecular Dynamics Simulation
}

\author{
Chedadi Oussama ${ }^{1(D)}$, El Aissouq Abdellah ${ }^{2 *} \cdot(\mathbb{D})$, El Ouardi Youssef ${ }^{3}$, , Bouachrine Mohammed ${ }^{4(D)}$, \\ Ouammou Abdelkrim ${ }^{1}$ iD
}

1 LIMOME Laboratory, Faculty of Sciences Dhar El Mahraz, Sidi Mohamed Ben Abdellah University, Fez, Morocco

2 Laboratory of Processes, Materials and Environment (LPME), Faculty of Science and Technology, Sidi Mohamed Ben Abdellah University, Fez, Morocco

3 Laboratory of Separation Technology, Lappeenranta University of Technology, Lappeenranta, Finland

4 MCNS Laboratory, Faculty of Sciences, Moulay Ismail University, Meknes, Morocco

* Correspondence: abbdellah.elaissouq@usmba.ac.ma (E.A.A.);

Scopus Author ID 57218453948

Received: 26.07.2021; Revised: 10.09.2021; Accepted: 16.09.2021; Published: 17.10.2021

\begin{abstract}
The Chymotrypsin-like protease $\left(3 \mathrm{CL}_{\mathrm{pro}}\right)$ is a drug target in the coronavirus because of its role in processing the polyproteins that are translated from the viral RNA. This study applied 3D quantitative structure-activity relationship (3D-QSAR), molecular docking, and ADMET prediction on a series of SARS-CoV 3CL pro inhibitors. The 3D-QSAR study was applied using Comparative Molecular Field Analysis (CoMFA) and Comparative Molecular Similarity Indices Analysis (CoMSIA) methods, which gave the cross-validation coefficient $\left(\mathrm{Q}^{2}\right)$ values of 0.64 and 0.80 , the determination coefficient $\left(\mathrm{R}^{2}\right)$ values of 0.998 and 0.993 and the standard error of the estimate (SEE) values of 0.046 and 0.091 , respectively. The acceptable values of the determination coefficient ( $\mathrm{R}^{2}$ test) to CoMFA and CoMSIA respectively corresponding to values of 0.725 and 0.690 utilizing a test set of seven molecules prove the high predictive ability of this model. Molecular docking analysis was utilized to validate 3DQSAR methods and explain the binding site interactions and affinity between the most active ligands and the SARS-CoV 3CLpro receptor. Based on these results, a novel series of compounds were predicted, and their pharmacokinetic properties were verified using drug-likeness and ADMET prediction. Finally, the best-docked candidate molecules were subjected to molecular dynamics (MD) simulation to affirm their dynamic behavior and stability.
\end{abstract}

Keywords: 3D-QSAR; SARS-CoV 3CL pro; molecular docking; ADMET prediction; MD simulation.

(C) 2021 by the authors. This article is an open-access article distributed under the terms and conditions of the Creative Commons Attribution (CC BY) license (https://creativecommons.org/licenses/by/4.0/).

\section{Introduction}

Severe acute respiratory syndrome (SARS) is a disease caused by the coronavirus (SARS-CoV), which highly infects the respiratory tract [1]. The SRAS-CoV disease starts with a Flu-like illness that evolves into respiratory failure followed by pneumonia, and in severe cases, by death. Therefore, the mortality rate of SARS is relatively higher compared to that of the common infections of the respiratory tracts and influenza [2].

The propagation of the SARS-CoV is said to originate from an animal's virus, most likely of bats, which then spread to other animals. The first SRAS-CoV infection in humankind was recorded in Sothern, China, in Guangdong in 2002 [3]. Starting from March 2003, the viral infection rapidly spread to other countries, causing 8000 infections and 800 deaths [4]. In 2004, 
no respiratory infection was documented in any part of the world [5,6]. Ever since then, a few cases of viral infection emerged from transmission from animals to humankind or because of laboratory accidents. Recent research shows that CoV-SARS can also be transmitted from one person to another $[7,8]$.

Following the clinical diagnosis of diverse patients who were infected by the viral infection SARS-CoV, common symptoms were recorded, including fever, diarrhea, shivering, myalgia, and malaise. None of these symptoms directly linked with the SARS diagnosis, even if the fever was the most commonly reported amongst patients, particularly those with chronic diseases and the elderly [9]. In most cases, the chronology of the viral infection's symptoms is manifested as diarrhea, shortness of breath, and cough. In extreme cases, the infection can evolve into respiratory distress requiring intensive care [10,11]. SARS is caused by a new human coronavirus (SARS-CoV), which encodes many viral proteases that proteolyze polyproteins to produce functional proteins. The major protease (Mpro), also known as the dimeric chymotrypsin-like protease (3CLpro), is one such highly conserved cysteine protease [12].

In this work, we focused on a series of SARS-CoV 3CLpro inhibitors to perform a theoretical study by using 3D-QSAR [13], molecular Docking [14,15], absorption, distribution, metabolism, excretion, and toxicity (ADMET) prediction [16,17], and molecular dynamics (MD) simulation. The 3D-QSAR methods, including comparative molecular similarity indices analysis (CoMSIA) and comparative molecular field analysis (CoMFA), were used to understand the impact of hydrophobic, electrostatic, steric, hydrogen bonds donor, and hydrogen bond acceptor fields on the inhibitory activity [18,19]. Molecular docking analysis was also applied to validate 3D-QSAR models and to study the orientations along with the probable binding conformations of the composites interacting with the SARS-CoV 3CLpro receptor. Based on the results of 3D-QSAR and docking analysis, a series of compounds were predicted. Then, the pharmacokinetic properties of predicted compounds were analyzed by drug-likeness and ADMET prediction. The best-docked compounds were subjected to MD simulation to affirm the final candidate molecules' conformational space and binding stability [20].

\section{Materials and Methods}

\subsection{Data set analysis.}

Concerning the generation of 3D-QSAR models, the values of the biological activity (values IC50 $(\mu \mathrm{M})$ ) were used, and a set of data about the inhibitors 3CLpro of SARS-CoV was synthesized by Konno et al. [21]. The values IC50 were converted to pIC50 values [22] and were then utilized as the dependent variable of this experimental work.

Table 1. Data set compounds and activity.

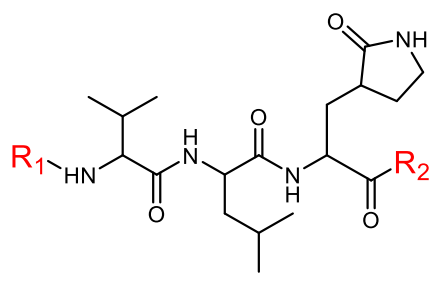

$(1-21)$<smiles>[R]NC(C(=O)NC([R2])C(=O)NC(Cc1cnc[nH]1)C(=O)c1nccs1)C(C)C</smiles>

(22-23) 


\begin{tabular}{|c|c|c|c|c|c|c|c|c|c|}
\hline No & R1 & R2 & $\begin{array}{l}\text { IC50 } \\
(\mu \mathrm{M})\end{array}$ & pIC50 & No & R1 & $\mathbf{R} 2$ & $\begin{array}{l}\text { IC50 } \\
(\mu \mathrm{M})\end{array}$ & pIC50 \\
\hline 1 & & & 36 & 4.444 & $1^{T}$ & & & 0.75 & 6.125 \\
\hline 2 & & & 85 & 4.071 & 14 & & & 1.2 & 5.921 \\
\hline 3 & & & 250 & 3.602 & 15 & & & 0.65 & 6.187 \\
\hline 4 & & & 280 & 3.553 & $16^{\mathrm{T}}$ & & & 1.7 & 5.770 \\
\hline 5 & & & 13 & 4.886 & 17 & & & 3.4 & 5.469 \\
\hline $6^{\mathrm{T}}$ & & & 24 & 4.620 & 18 & & & 2.9 & 5.538 \\
\hline 7 & & & 6.8 & 5.168 & 19 & & & 1.5 & 5.824 \\
\hline 8 & & & 2 & 5.699 & 20 & & & 7.5 & 5.125 \\
\hline 9 & & & 1.7 & 5.769 & 21 & & & 9.5 & 5.023 \\
\hline 10 & & & 2.3 & 5.638 & 22 & & & 260 & 3.585 \\
\hline 11 & & & 0.92 & 6.036 & $23^{T}$ & & & 210 & 3.678 \\
\hline 12 & & & 1.9 & 5.721 & & & & & \\
\hline
\end{tabular}

\subsection{The study of $3 D-Q S A R$ model.}

\subsubsection{Alignment and optimization of the molecular database.}

With the help of the program package SYBYL-X 2.1, the design, molecular optimization, and alignment were implemented for the 3D-QSAR study [23]. This program utilizes the geometrical optimization of the Tripos Force Field [24], which is based on the following criteria:

- $0.001 \mathrm{kcal} / \mathrm{mol} \AA$ A convergence criterion.

- 10000 iterations as a maximum number.

- The model Gasteiger-Hücke is utilized to calculate the atomic charges [25].

\subsubsection{CoMFA and CoMSIA procedures.}

The method of CoMFA [26,27] lies within the alignment of the different compounds in a grid lattice of $2.0 \AA$ spacing. Concerning the probation of the electrostatic (Coulombic) and 
the steric (Lennard-Jones) field energies, an sp3 carbon atom was utilized with a +1 charge. The values of the interactions' cutoff energies must follow the order of $30 \mathrm{kcal} / \mathrm{mol}$. Column filtering based on the calculation of the molecular-field energies with a variation less than 2.0 $\mathrm{kcal} / \mathrm{mol}$ was applied to diminish the computation time without impacting the CoMFA models' quality [16].

The CoMSIA method [28] is a prolongation of the CoMFA method. In the study of CoMSIA, hydrophobic, electrostatic, steric, hydrogen bond acceptor, and donor fields were calculated on-grid lattice of a $2.0 \AA$. The values of the interactions' cutoff energies must follow the order of $30 \mathrm{kcal} / \mathrm{mol}$ [28], and the attenuation factor was put to the value of 0.3 . Column filtering is based on calculating the molecular-field energies with a variation of less than 2.0 $\mathrm{kcal} / \mathrm{mol}$.

The CoMFA and CoMSIA method rely on the robustness of the partial-least-squares (PLS) [29] analysis which was treated by the cross-validation method through the use of the procedure leave-one-out (LOO) [30]. For the 3D-QSAR to be statistically predictable and credible, the values of the regression coefficient $\mathrm{Q}^{2}$ of the cross-validation and the values of the regression coefficient $R^{2}$ need to be greater than 0.5 and 0.6 , respectively. For the model of CoMFA and CoMSIA to be absolutely reliable, it is mandatory that the Fisher coefficient and the standard error of estimation (SEE) of $\mathrm{R}^{2}$ be calculated using PLS with a non-cross validation method. The prediction ability of the chosen model was verified by using the external validation (test set) $[31,32]$.

\subsection{Docking study.}

Molecular docking permits to detection and visualization of the potential interactions between the ligands and the receptor (SARS-CoV 3CL pro). Concerning the necessary steps to realize the docking analyses, the adequate protein SARS-CoV $3 \mathrm{CL}_{\text {pro }}$ starting from the Data Bank Protein (PDB code: 1WOF) [33] was being downloaded, the water molecules, as well as the original ligand, were eliminated, and the active site of the protein was detected using the AutoDock tools [34] by defining the cubic grid box $44 \AA$ x $46 \AA$ x $58 \AA$ and $40 \AA$ x $54 \AA$ x 40 $\AA$ at $0.375 \AA$

Finally, the most active molecules and the most potent inhibitors were docked by using AutoDock 4.2 [35] in the active zone of the protein 1WOF, and the results are visualized utilizing PyMol [36] et Discovery Studio 2017 software's [37].

\subsection{Molecular dynamics simulation.}

After performing the molecular docking study, the best-scored complexes of SARSCOV (PDB code: 1WOF) were then subjected to molecular dynamics simulations. All the calculations were performed using the GROMACS version (2020.1-1) [38]. The topology file of each compound was created by the CHARMM General Force Field (CGenFF) server [39], while the protein topology was created by 'pdb2gmx' script. The simulations were run using the CHARMM36 all-atom (March 2019) force field [40] in a triclinic box with a distance of $1.0 \mathrm{~nm}$ and a TIP3P water model solvated system [41]. The neutralization of the system was performed by adding sodium and chloride ( $\mathrm{Na}+\mathrm{Cl}-)$ ions. The energy minimization of the system was subjected to 50,000 steps using the steepest descent algorithm. Then, the production of MD simulation was run for $20 \mathrm{~ns}$ for each simulation at a temperature of $300 \mathrm{k}$, a pressure of 1 bar, and a time step of 2 fs. 


\section{Results and Discussion}

\subsection{D-QSAR analysis.}

\subsubsection{Molecular alignment.}

Thanks to the compilation of the various compounds of the training and test sets in the module of distilling off the Sybyl software, it was possible to visualize the common structure (Core).
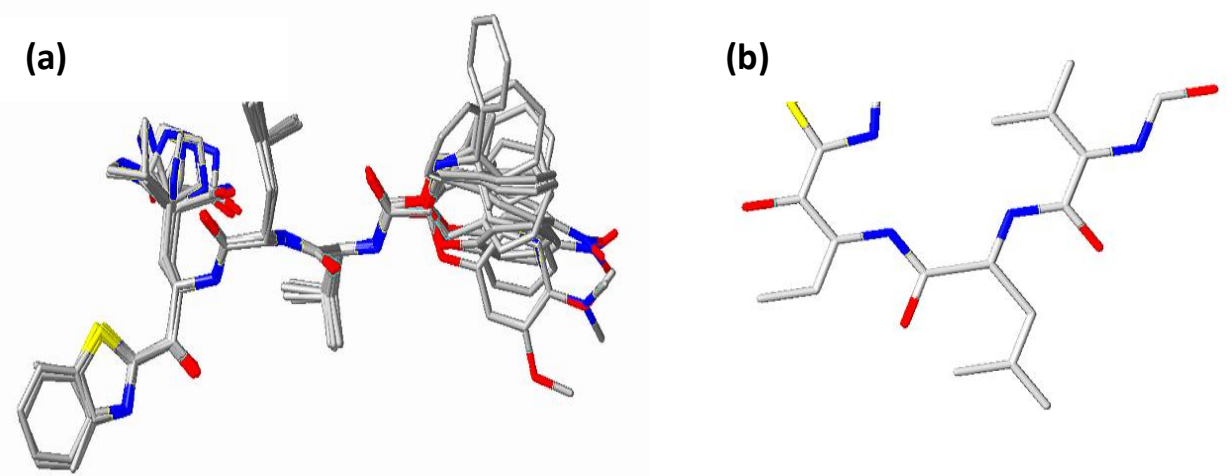

Figure 1. Molecular alignment of all molecules in the data set: (a) ligand-based alignment model of all the compounds. (b) Molecular core.

\subsubsection{CoMFA and CoMSIA analysis.}

The compilation of the CoMSIA and CoMFA system database resulted in itemized detailed statistical results to better understand the influence of electrostatic, steric, hydrophobic, hydrogen bond acceptor, and hydrogen bond donor fiends on the inhibitory activity of SARS-CoV 3CLpro in Table 2.

Table 2. Analytical results of CoMSIA and CoMFA models using PLS statistics.

\begin{tabular}{|c|c|c|c|c|c|c|c|c|c|c|c|}
\hline \multirow{2}{*}{ 3D-QSAR } & \multirow{2}{*}{$Q^{2} \mathrm{cv}$} & \multirow{2}{*}{$\mathbf{r}^{2}$ ncv } & \multirow{2}{*}{ SEE } & \multirow{2}{*}{$\mathbf{N}$} & \multirow{2}{*}{$\mathbf{F}$} & \multirow{2}{*}{ Pred_r ${ }^{2}$} & \multicolumn{5}{|c|}{ Relative $\%$ contributions } \\
\hline & & & & & & & $S$ & $E$ & $\mathrm{H}$ & $D$ & $A$ \\
\hline CoMFA & 0.64 & 0.998 & 0.046 & 5 & 1462.69 & 0.725 & 64.4 & 35.6 & - & - & - \\
\hline CoMSIA & 0.80 & 0.993 & 0.091 & 5 & 371.624 & 0.690 & 26.7 & - & 73.3 & - & - \\
\hline
\end{tabular}

Q2: Cross-validated determination coefficient; F = Fischer value; $\mathrm{N}$ : Optimum number of components obtained from cross-validated PLS analysis and same used in the final non-cross-validated analysis; R2: Non-crossvalidated determination coefficient; R2 test: External validation determination coefficient; SEE: Standard error of the estimate.

The results of the CoMFA model revealed the determination coefficient $\left(\mathrm{R}^{2}\right)$ value of 0.998, the cross-validated determination coefficient $\left(\mathrm{Q}^{2}\right)$ value 0.64 , and the test set determination coefficient $\left(\mathrm{R}_{\text {test }}^{2}\right)$ value of 0.75 . The observed values of pIC50 and their residues which the CoMFA model predicted are listed in Table 3. Figure 2 shows the correlation between the predicted and observed values of pIC50 obtained by the CoMFA model.

In the model CoMSIA, the ideal number of the principal components utilized to generate this model is 5 . The principal factors of activity control are the hydrophobic and the steric fields. The generated model showed a non-cross-validated correlational coefficient $\left(\mathrm{R}^{2}{ }_{\mathrm{ncv}}\right)$ and a cross-validated determination coefficient $\left(\mathrm{Q}^{2}{ }_{\mathrm{cv}}\right)$ values of 0.993 and 0.80 , respectively. 


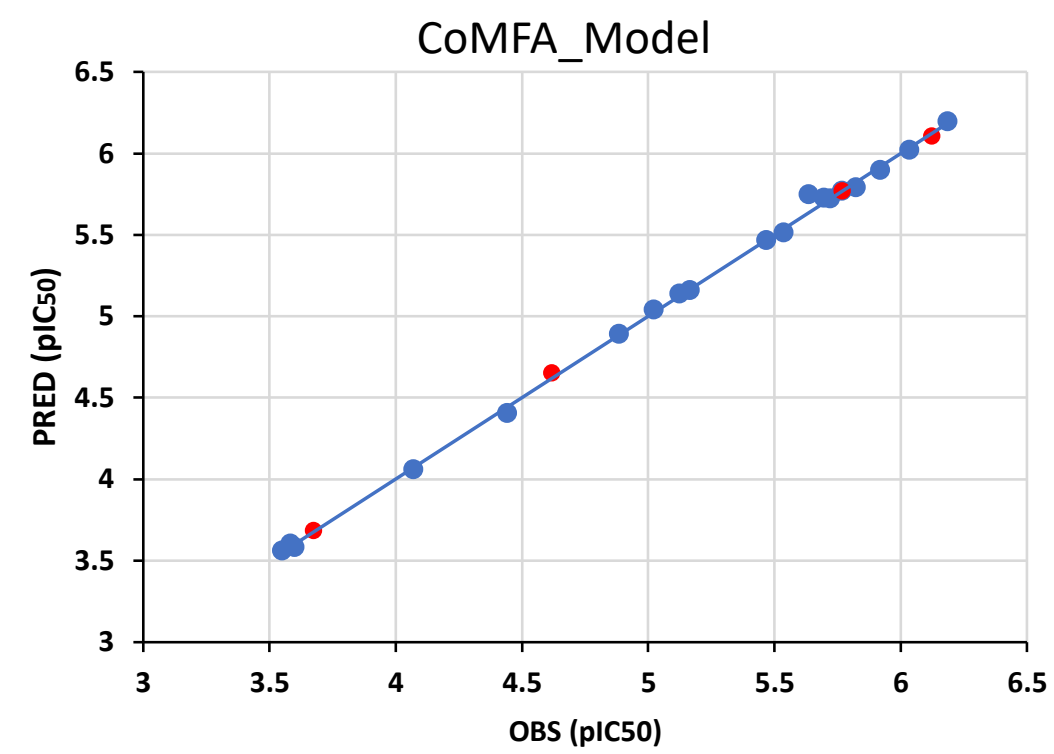

Figure 2. Correlations between experimental observed (pIC50) and predicted (PIC50) ones for the molecules of the test set (red color) and the training set (blue color) using the CoMFA model.

The value of the $\mathrm{R}_{\text {test }}^{2}$ is 0.691 , indicating a good predictive ability of the generated model. The analysis of these statistical parameters indicates good stability of the model CoMFA and a credible prediction capability. The observed and predicted values of pIC50 and their corresponding residues are listed in Table 3. Figure 3 shows the correlations between the observed and predicted values of pIC50 obtained by the CoMSIA model.

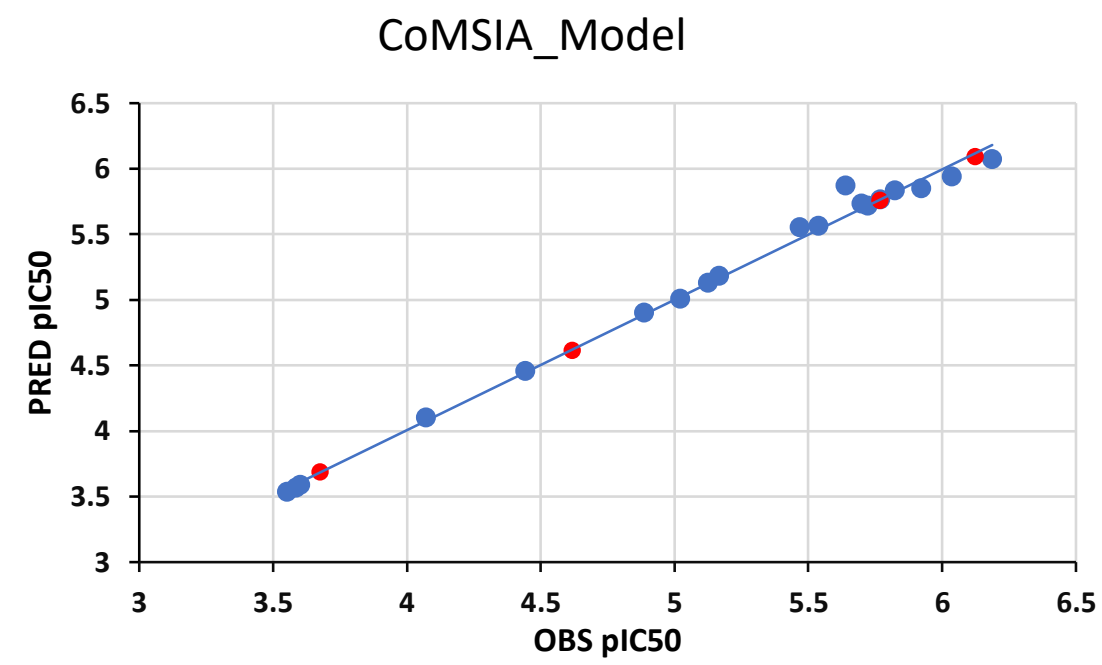

Figure 3. Correlations between experimental activities (pIC50) and predicted (pIC50) ones for the molecules of the test set (red color) and the training set (blue color) using the CoMSIA model.

Table 3. The values of the predicted and observed activities and the residual values obtained by 3D QSAR (CoMFA and CoMSIA).

\begin{tabular}{c|c|c|c|c|c} 
No & pIC50 & pIC50_CoMFA & Residual & pIC50_CoMSIA & Residual \\
\hline 1 & 4.4437 & 4.404 & 0.0397 & 4.456 & -0.0123 \\
\hline 2 & 4.0706 & 4.06 & 0.0106 & 4.097 & -0.0264 \\
\hline 3 & 3.6021 & 3.582 & 0.0201 & 3.586 & 0.0161 \\
\hline 4 & 3.5528 & 3.56 & -0.0072 & 3.53 & 0.0228 \\
\hline 5 & 4.8861 & 4.888 & -0.0019 & 4.896 & -0.0099 \\
\hline $6^{\mathbf{T}}$ & 4.6198 & 4.65 & -0.0302 & 4.609 & 0.0108 \\
\hline 7 & 5.1675 & 5.157 & 0.0105 & 5.181 & -0.0135
\end{tabular}




\begin{tabular}{c|c|c|c|c|c} 
No & pIC50 & pIC50_CoMFA & Residual & pIC50_CoMSIA & Residual \\
\hline 8 & 5.699 & 5.724 & -0.025 & 5.732 & -0.033 \\
\hline 9 & 5.7696 & 5.768 & 0.0016 & 5.753 & 0.0166 \\
\hline 10 & 5.6382 & 5.745 & -1.3013 & 5.87 & -0.2317 \\
\hline 11 & 6.0362 & 6.02 & -1.5763 & 5.936 & 0.1002 \\
\hline 12 & 5.7212 & 5.719 & -1.2753 & 5.715 & 0.0062 \\
\hline $13^{\mathbf{T}}$ & 6.124 & 6.102 & -1.6583 & 6.089 & 0.0359 \\
\hline 14 & 5.9208 & 5.895 & -1.4513 & 5.848 & 0.0728 \\
\hline 15 & 6.1870 & 6.193 & -1.7493 & 6.069 & 0.1181 \\
\hline $16^{\mathbf{T}}$ & 5.7695 & 5.764 & -1.3203 & 5.764 & 0.0056 \\
\hline 17 & 5.4685 & 5.464 & 0.0045 & 5.548 & -0.0795 \\
\hline 18 & 5.5376 & 5.512 & 0.0256 & 5.56 & -0.0224 \\
\hline 19 & 5.8239 & 5.787 & 0.0369 & 5.832 & -0.0081 \\
\hline 20 & 5.1249 & 5.135 & -0.0101 & 5.124 & 0.0009 \\
\hline 21 & 5.0223 & 5.037 & -0.0147 & 5.005 & 0.0173 \\
\hline 22 & 3.585 & 3.603 & -0.018 & 3.566 & 0.019 \\
\hline $23^{\mathbf{T}}$ & 3.6778 & 3.682 & -0.0042 & 3.683 & -0.0052 \\
& & $\mathbf{T}$ Represents the test set compounds. &
\end{tabular}

As a result, the steric (64.4\% in the CoMFA model and 26.7\% in CoMSIA model) and hydrophobic ( $73.3 \%$ in CoMSIA model) fields play an important role in controlling the activity. Also, the comparative results of CoMFA and CoMSIA models indicate that the results obtained by the CoMSIA study are significant and can be used to predict new SARS-CoV $3 \mathrm{CL}_{\text {pro }}$ inhibitors with high potency activity.

\subsubsection{CoMFA and CoMSIA graphical interpretation.}

The contour maps of CoMSIA and CoMFA methods allow visualizing the regions in which the activity raises or diminishes in the referential molecular structure (Compound 15); after the compilation of the model, it was permitted to see the electrostatic and steric contour maps and Hydrophobic and Steric contour maps of CoMFA and CoMSIA, respectively. All the contours symbolized the $20 \%$ and $80 \%$ default contributions level, respectively, for disfavored and favored regions.

The CoMFA steric and electrostatic contour maps are presented in Figure 4. Hydrophobic and steric contour maps of CoMSIA are revealed in Figure 5.

\subsubsection{CoMFA contour maps.}

On the one hand, the electrostatic interactions of CoMFA are characterized by the red contour, which marks areas of increased activity with negative charges, and the blue contour, which marks areas where positive charges are favored.
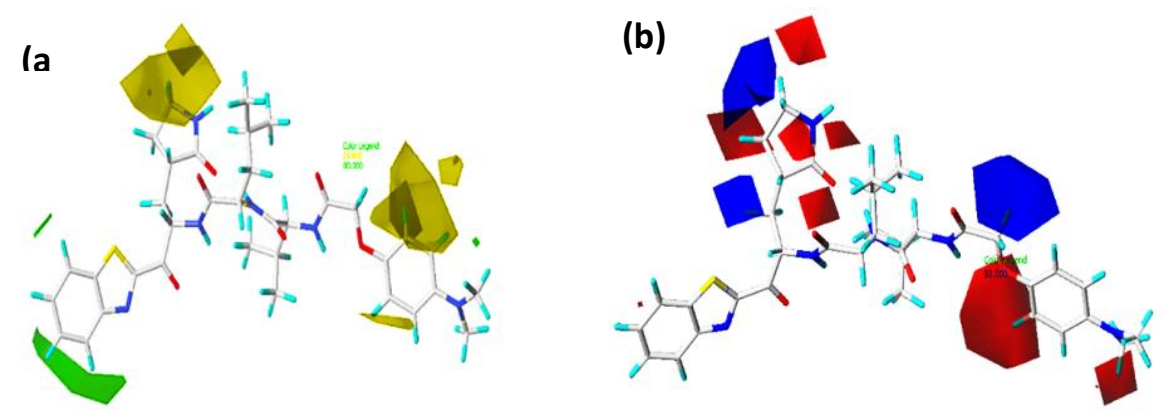

Figure 4. Standardized coefficient Contour maps of CoMFA analysis of the SARS-CoV 3CL $L_{\text {pro }}$ inhibitors activity (compound 15): (a) steric Contour maps; (b) electrostatic Contour maps. 
On the other hand, the steric interactions of CoMFA are characterized by the yellow contour where the cumbersome substituents are unfavored and the green contour where these latter are favored Figures 4 (a) and (b).

The contour map, which is marked in green near the R1 position (Figure 4 (a)), suggests that the R1 group is favorable in terms of steric field, which could justify the reason why the compounds 1 to 6 , which contains $\mathrm{R}=4.5$ - dihydrothizole with $\mathrm{pIC}_{50}$ between 3.552 and 4.886 are the less active compounds than compounds 8 to 20 which contains $\mathrm{R}=$ benzothiazole with pIC50 between 5.124 and 6.187. Moreover, a larger unfavorable yellow contour near the position R1 and pyrrolidinone group indicates that bulky regions decrease activity.

The electrostatic contour map is portrayed in (Figure 4 (b)). Red contours indicate regions where negative groups increase the activity. A large red contour map around the R1 position indicates that the electronegative groups at this region may increase the activity. This may explain why compound 10 which contains $\mathrm{R}=2$-phenoxyacetaldehyde showed a significant decrease in activity (pIC50 $=5.638$ ) than compound 11 (pIC50 =6.036), which contains $\mathrm{R}=2$-(4-methoxyphenoxy) acetaldehyde. On the other hand, the region where a less negative potential electrostatic field (diminishes the activity of the inhibitor) is marked by the blue contour.

\subsubsection{CoMSIA contour maps.}

The best CoMSIA model was generated using hydrophobic and steric fields. Figures 5 (a) and (b) represent the steric and hydrophobic contour maps, respectively. Steric interactions are characterized by green (sterically-favorable) and yellow (sterically-unfavorable) colored contours, while hydrophobic interactions are characterized by blue (hydrophobicallyunfavorable) and magenta (hydrophobically-favorable) colored contours.

(a)

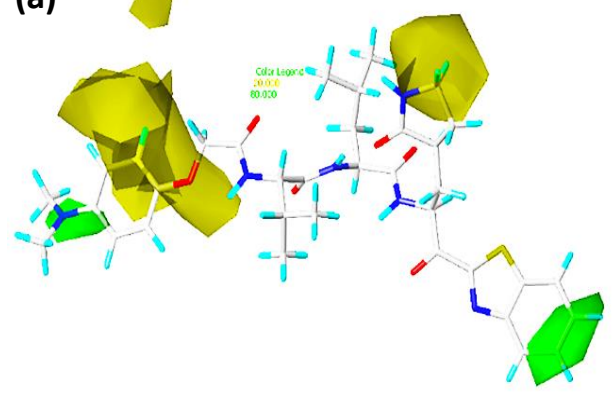

(b)

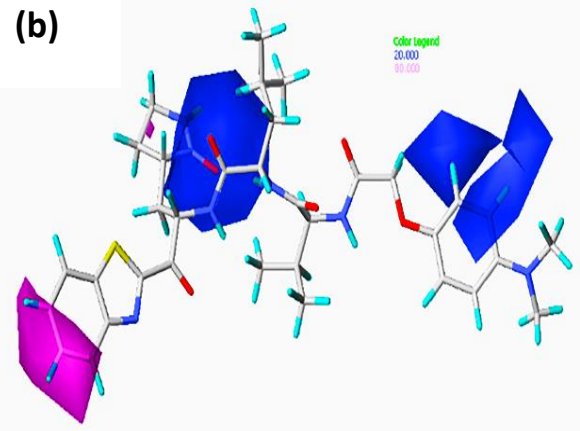

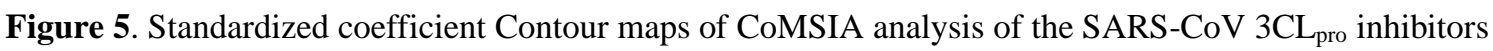
activity ( compound 15) : (a) Steric contour map, (b) Hydrophobic contour map.

Figure 5 (a) shows the CoMSIA steric contour map. As shown in this figure, a green contour map near the position R2 and phenyl group R1 suggests that the R2 group is favorable in terms of the steric field. This may justify why compounds 1 to 6 (pIC50 between 3.552 and 4.886) containing $\mathrm{R}=4.5$ - dihydrothizole are less active than compounds 8 to 20 (pIC50 between 5.124 and 6.187), which contain $\mathrm{R}=$ benzothiazole. Moreover, a larger unfavorable yellow contour near the position R1 and pyrrolidinone group indicates that bulky groups in these regions decrease the activity.

Figure 5 (b) shows the CoMSIA hydrophobic contour map. As described in this figure, the magenta contour around the R2 group reveals that hydrophobicity substituent at this 
position would increase activity. Moreover, a large blue area around the R1 and pyrrolidinone groups indicates that the hydrophobic groups in these positions may decrease the activity.

\subsection{Molecular docking results.}

In order to predict the binding site interactions and energy, molecular docking analysis was applied on the most potent inhibitor in the dataset. The native ligand was first re-docked in its PDB structure (PDB ID: 1WOF). The best-obtained pose gave the root-mean-square deviation (RMSD) value of 1.11, using 50 iterations. The superposition between co-crystallized and re-docked ligands is shown in Figure 6.

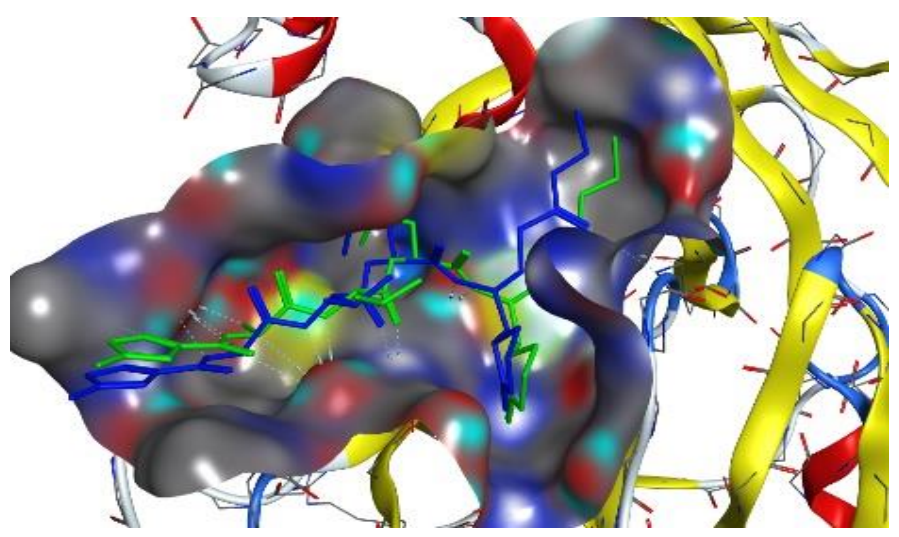

Figure 6. Superposition of the reference ligand (green stick) with the re-docked ligand (bleu stick) in the pocket site SARS-CoV 3CLpro (PDB ID: 1WOF).

After docking validation, the most potent inhibitor in the dataset (compound 15) was used to generate molecular docking analysis. The binding site interactions between compound 15 and SARS-CoV 3CLpro are shown in Figure 7. As described in this figure, Compound 15 forms 3 Van der Waals interactions with GYS 44, THR 25, and GLN 189 amino acids and 4 conventional Hydrogen bond interactions with LEU 141, CYS 145and GLU 166 amino acids. This type of interaction and mostly Hydrogen bond interactions explain why this compound shows high stability in the catalytic pocket of SARS-CoV 3CLpro $(-7.5 \mathrm{kcal} / \mathrm{mol})$.

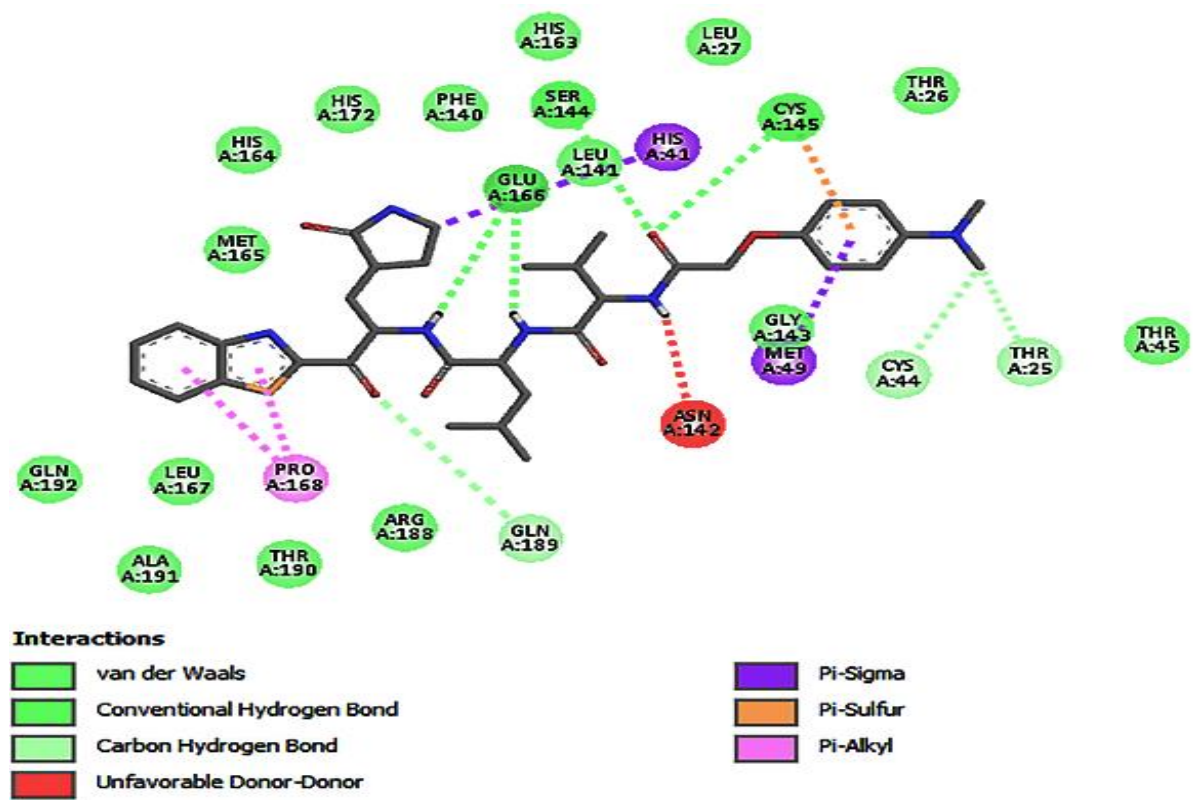

Figure 7. Docking results of compounds 15 with SARS-CoV 3CLpro protein (PDB code: 1WOF). 


\subsection{Design of new compounds.}

The 3D-QSAR (CoMSIA and CoMFA) model provided us with the needed information to elaborate a conceptual strategy of new inhibitors with enhanced effectiveness.

After the conceptualization of novel inhibitors' structure in accordance with the directive line of the QSAR model (Figure 8), the compounds are aligned and modeled in the study of CoMSIA. The results of the activities of the predicted compounds are presented in Table 4.

The activities' values of the predicted compounds show that they are very high compared to the most active compound in the basic database.

The final step is the realization of molecular docking of the new predicted compounds $P$ 1-5. The results of the interactions between the active site and the SARS-CoV 3CL pro protein (PDB ID: $1 \mathrm{WOF}$ ) are presented in Table 4. The results indicate that the binding energy of predicted compounds in the active site of SARS-CoV 3CLpro protein (PDB ID: 1WOF) is larger than the most potent. These docking results show that the chemical binding energies between the predicted compounds are lower than that of the most active molecule in the data set (compound 15). This reflects that these new compounds are more stable when binding with the active site of the SARS-CoV 3CL pro protein (PDB ID: 1WOF); therefore, it gives a high inhibitory effect.

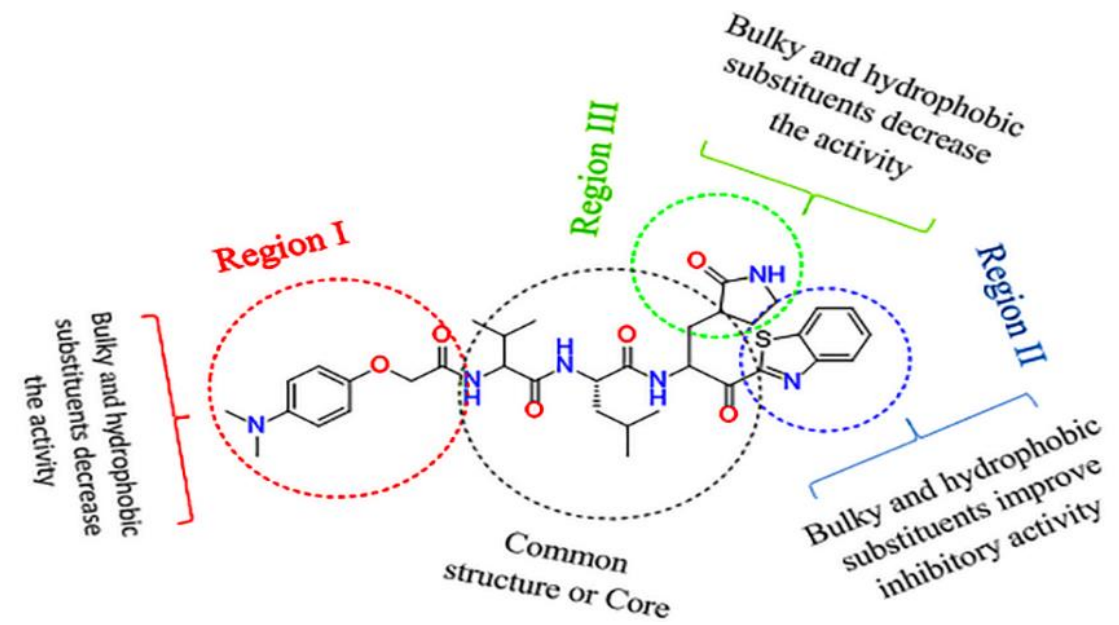

Figure 8. Structure-activity relationship (SAR) information obtained from the 3D-QSAR study.

Table 4. Structures of newly designed compounds and their corresponding predicted pIC50 values and binding free energy.

\begin{tabular}{ll|l|l|l|} 
Compounds & CoMSIA_pred & Binding energy (kcal/mol) \\
\hline &
\end{tabular}




\begin{tabular}{l|l|l|l} 
Compounds & Structures & CoMSIA_pred & Binding energy (kcal/mol) \\
\hline &
\end{tabular}

(a)

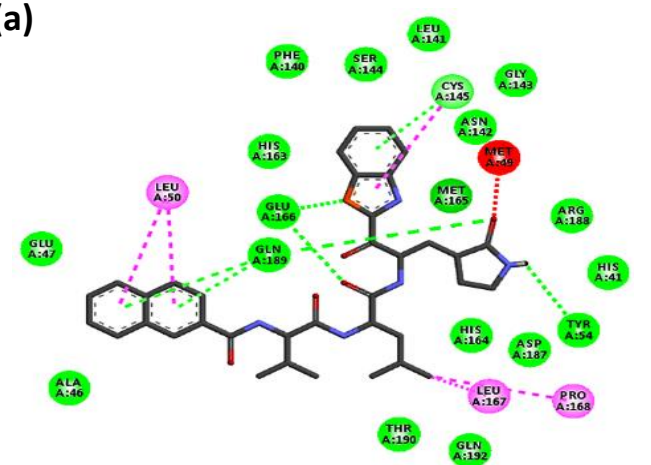

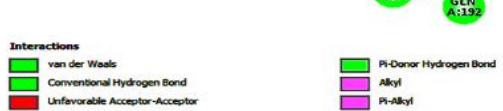

(b)

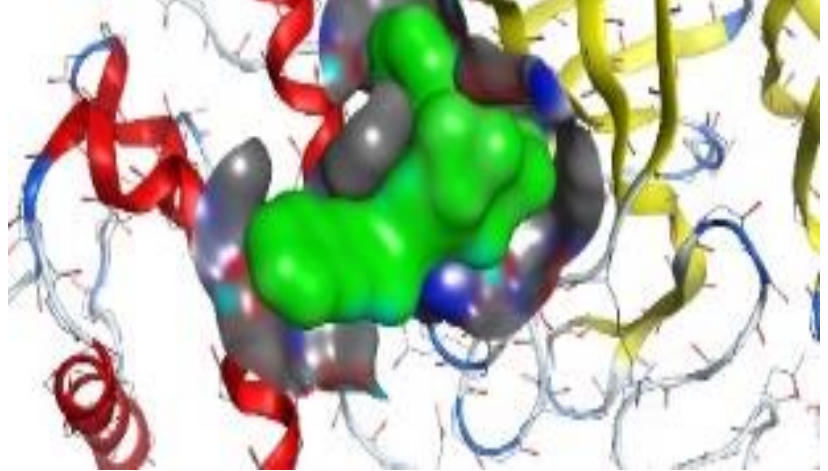

Figure 9. Molecular docking of compound P1 with SARS-CoV $3 \mathrm{CL}_{\text {pro }}$ protein. (a) Binding site interactions of $2 \mathrm{D}$ view, (b) the binding conformation of $3 \mathrm{D}$ view.

(a)

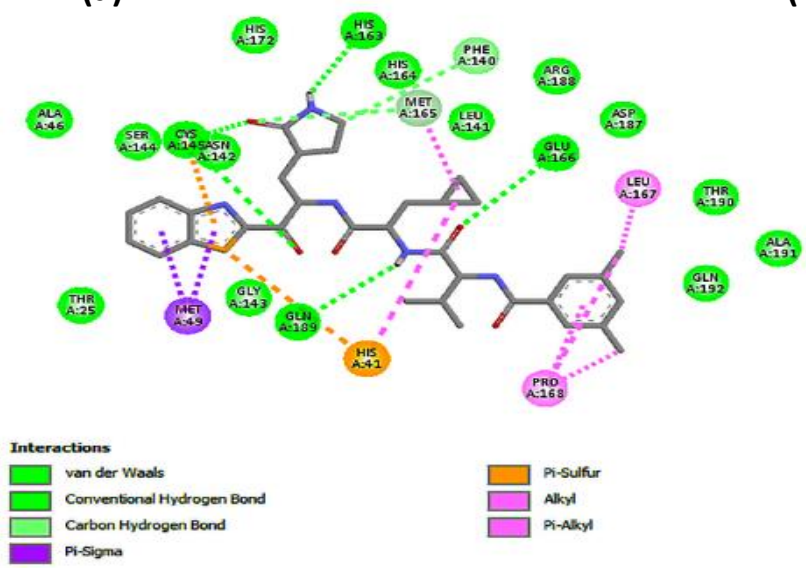

(b)

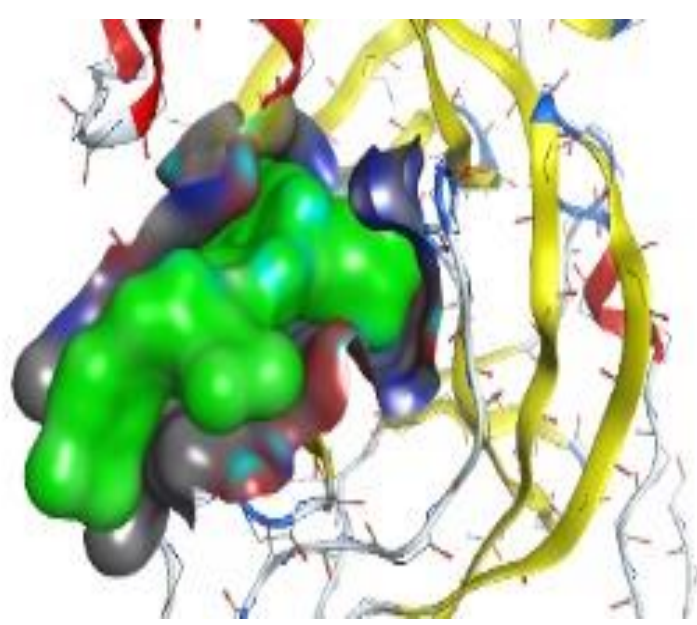

Figure 10. Molecular docking of compound $P 2$ with SARS-CoV 3CL pro protein. (a) Binding site interactions of $2 \mathrm{D}$ view, (b) binding conformation of $3 \mathrm{D}$ view.

(a)

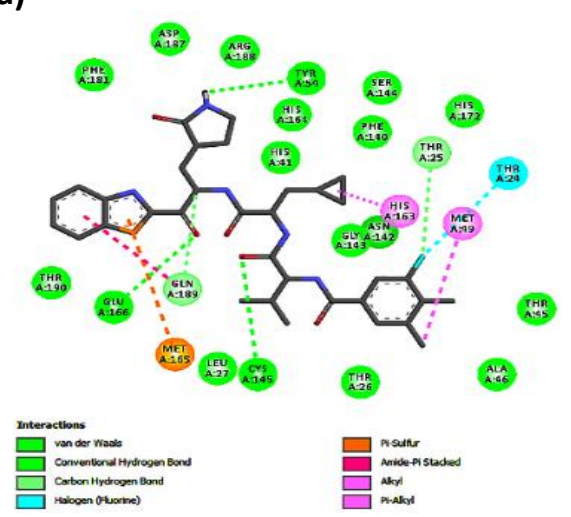

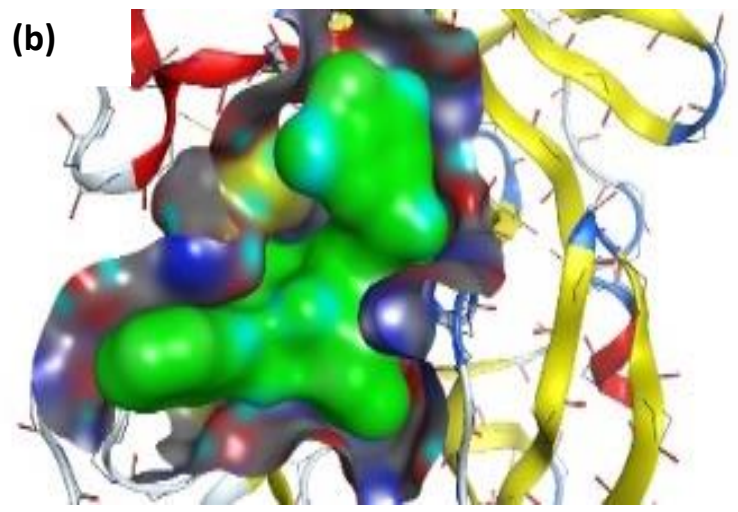

Figure 11. Molecular docking of compound $\mathrm{P} 3$ with SARS-CoV $3 \mathrm{CL}_{\text {pro }}$ protein. (a) Binding site interactions of $2 \mathrm{D}$ view, (b) binding conformation of $3 \mathrm{D}$ view. 
The molecular docking results of the predicted highly active molecules P1, P2, and P3 with the receptor 3CLpro du SARS-CoV showed adhesion of the protein's active site through hydrogenic and hydrophobic liaisons, which explains the affinity of these structures in accordance with the protein SARS-CoV 3CLpro. See the diagrams in 2D and 3D of the ligandreceptor interactions in Figures 9-11.

\subsection{Drug- likeness and ADMET prediction.}

Before the experiment, it is necessary to perform the drug-likeness and the pharmacokinetic properties of novel predicted compounds [42,43]. Here, the drug-likeness and the absorption, distribution, metabolism, excretion, and toxicity (ADMET) were calculated using the pkCSM [44] and SwissADMET [45] webservers. The results are listed in Table 5.

Table 5. In silico prediction of drug-likeness and ADMET properties.

\begin{tabular}{|c|c|c|c|c|c|c|c|c|c|c|c|c|c|c|}
\hline \multirow[b]{2}{*}{ No. } & \multirow[b]{2}{*}{$\begin{array}{c}\text { MW } \\
\left(\mathrm{g} / \mathrm{mol}^{\mathbf{1}}\right)\end{array}$} & \multirow[b]{2}{*}{ HBA } & \multirow[b]{2}{*}{ HBD } & \multirow[b]{2}{*}{$\begin{array}{c}\text { TPSA } \\
\left(\mathbf{A}^{2}\right)\end{array}$} & \multirow[b]{2}{*}{$\log P$} & \multirow[b]{2}{*}{$\log S$} & \multirow[b]{2}{*}{ HIA } & \multirow[b]{2}{*}{ BBB } & \multirow[b]{2}{*}{$\begin{array}{c}\text { CYP 2D6 } \\
\text { inhibition }\end{array}$} & \multirow[b]{2}{*}{$\begin{array}{c}\text { Total } \\
\text { Clearance }\end{array}$} & \multicolumn{2}{|c|}{ Toxicity } & \multicolumn{2}{|c|}{ Drug-likeness } \\
\hline & & & & & & & & & & & AMES & $\begin{array}{c}\text { Skin } \\
\text { Sensitization }\end{array}$ & Lipinski & $\begin{array}{c}\text { Bioavailability } \\
\text { Score }\end{array}$ \\
\hline P1 & 655.81 & 6 & 4 & 174.60 & 4.62 & -4.22 & 79.37 & -1.41 & No & -0.198 & No & No & Yes & 0.55 \\
\hline $\mathrm{P} 2$ & 672.635 & 7 & 4 & 174.60 & 4.53 & -4.75 & 78.60 & -1.56 & No & -0.254 & No & No & Yes & 0.55 \\
\hline P3 & 670.19 & 7 & 4 & 174.60 & 4.33 & -4.31 & 79.17 & -1.59 & No & -0.251 & No & No & Yes & 0.55 \\
\hline 15 & 677.868 & 9 & 3 & 175.04 & 4.39 & -4.13 & 72.159 & -1.60 & No & -0.073 & No & No & No & 0.17 \\
\hline
\end{tabular}

$\mathrm{MW}=$ molecular weight, $\mathrm{HBA}=$ number of hydrogen bond acceptor, $\mathrm{HBD}=$ number of hydrogen bond donor,

TPSA = topological polar surface area, $\log \mathrm{P}=$ octanol-water partition coefficient, $\log \mathrm{s}=$ Water solubility,

$\mathrm{HIA}=$ human intestinal absorption, $\mathrm{BBB}=$ blood brain barrier.

As shown in Table 5, all predicted compounds exhibit a moderate ADMET parameter. The high values of human intestinal absorption (HIA > 30\%) indicate that the predicted compounds exabit a moderate permeation across the intestinal membrane. The $\log \mathrm{P}$ and $\log \mathrm{S}$ values of all compounds are in the best range, which means they had good absorbency and good solubility, respectively. None of the predicted compounds were found to be a potent inhibitor of CYP450 2D6. Also, the test of AMES and Skin Sensitization revealed that the predicted compounds are harmless. In summary, it can be concluded that the predicted compounds are likely to be orally active.

\subsection{Molecular dynamics simulation.}

In order to validate the results of molecular docking and affirm the stability of docked compounds in the binding pocket of SARS-CoV $3 \mathrm{CL}_{\text {pro }}$ protein, compounds 15 and $\mathrm{P} 1$ were subjected to MD simulation. All the systems were employed for 20 ns time scale simulation. The Root Mean Square Deviation (RMSD) of 1WOF_M15 and 1WOF_P1 complexes are given in Figure 10 (a). This figure shows that the average RMSD value of 1WOF_M15 and 1WOF_P1 systems are 0.31 and $0.23 \mathrm{~nm}$, respectively. These results indicate that the 1WOF_P1 complex is more stable than the 1WOF_M15 complex during the simulations. The Root Means Square Fluctuation (RMSF) of both complexes and free 6LU7 protein is also shown in Figure 10 (b). This parameter is an indicator of residual flexibility [46-48]. From the results of the RMSF value, we conclude that the P3_6LU7 complex shows less conformational 
changes during the simulations except for the C-terminal end (Figure 10 (b)). All of these results are in agreement with that obtained by $3 \mathrm{D}-\mathrm{QSAR}$ and molecular docking.

RMSD

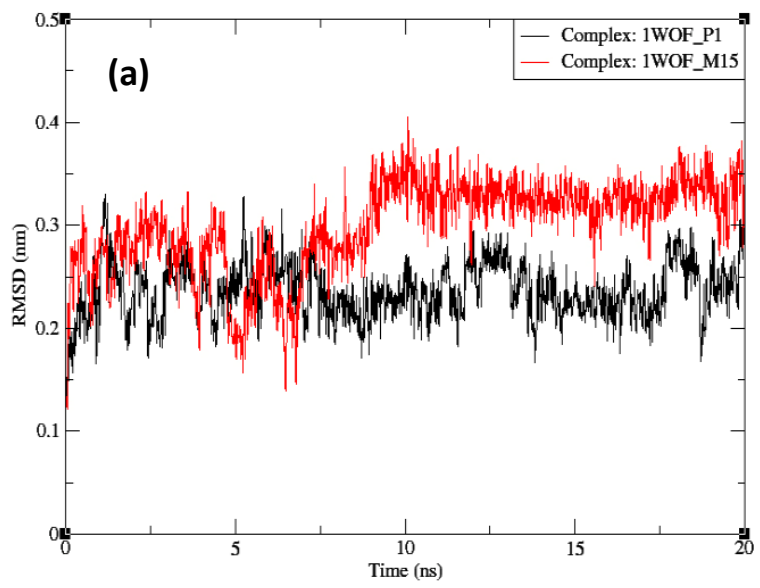

RMS fluctuation

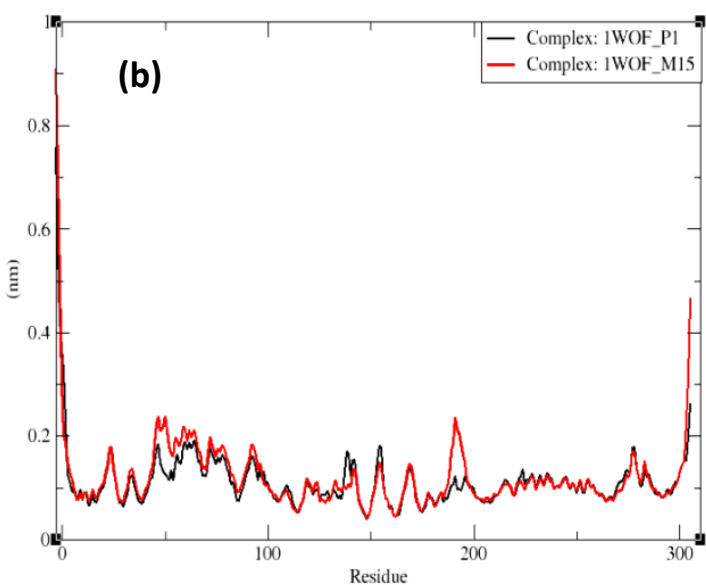

Figure 10. Molecular dynamics simulations. (a) RMSD of backbone over the $20 \mathrm{Ns}$ of MD simulation at $300 \mathrm{~K}$ and 1 bar, (b) RMSF of residues during MD simulation. In all Figures, the red color represents the 1WOF_M15 complex, and the black color represents the 1WOF_P1 complex.

\section{Conclusions}

This study proposes new inhibitor compounds of powerful SARS-CoV 3CL pro inhibitors from a series of the compounds through the application of 3D-QSAR studies, including CoMFA and CoMSIA.

The results of CoMFA and CoMSIA studies (PLS method of the regression of the smallest partial squares) show that the obtained values of $\mathrm{Q}^{2}, \mathrm{R}^{2}$ test, and $\mathrm{R}^{2}$ are very high and elevated for all models. The study of the chemical interactions of the binding site between the ligands and the $1 \mathrm{WOF}$ protein is carried out using molecular docking. According to the docking results, it can be inferred that there is a synergy with the 3D-QSAR studies.

Based on these studies, four new compounds, P1-5, were predicted using the CoMSIA method. The results of the docking analysis show that compared to the most potent inhibitor in the data set (composed 15), the predicted compounds have a high degree of stability in the binding pocket of the SARS-CoV 3CL pro receptor, and their pharmacokinetic properties were verified by drug-likeness and ADMET prediction. The results of MD simulation also indicate that the compound $\mathrm{P} 1$ is more stable in the binding pocket of the SARS-CoV 3CLpro receptor during the $20 \mathrm{~ns}$ of the simulation time.

\section{Funding}

This research received no external funding.

\section{Acknowledgments}

The authors want to thank the Moroccan Association of Theoretical Chemistry (MATC) for its relevant help regarding the software.

\section{Conflicts of Interest}

All authors declare that they have no conflict of interest in this work. 


\section{References}

1. Chiu, R.W.K.; Jin, Y.; Chung, G.T.Y.; Lui, W.; Chan, A.T.C.; Lim, W.; Lo, Y.M.D. Automated extraction protocol for quantification of SARS-Coronavirus RNA in serum: an evaluation study. BMC Infectious diseases 2006, 6, 20, https://doi.org/10.1186/1471-2334-6-20.

2. Ezhilan, M.; Suresh, I.; Nesakumar, N. SARS-CoV, MERS-CoV and SARS-CoV-2: A Diagnostic Challenge. Measurement 2021, 168, 108335, https://doi.org/10.1016/j.measurement.2020.108335.

3. Zhang, J.; Wang, D.; Li, Y.; Zhao, Q.; Huang, A.; Zheng, J.; Chen, W. SARS coronavirus nucleocapsid protein monoclonal antibodies developed using a prokaryotic expressed protein. Hybridoma 2011, 30, 481-485, https://doi.org/10.1089/hyb.2011.0028.

4. Weinstein, R.A. Planning for Epidemics — The Lessons of SARS. N Engl J Med 2004, 350, 2332-2334, https://doi.org/10.1056/nejmp048082.

5. Hourfar, M.K.; Roth, W.K.; Seifried, E.; Schmidt, M. Comparison of Two Real-Time Quantitative Assays for Detection of Severe Acute Respiratory Syndrome Coronavirus. J. Clin. Microbiol. 2004, 42, 20942100, https://doi.org/10.1128/jcm.42.5.2094-2100.2004.

6. Lal, S.K. (ed.) Molecular biology of the SARS-coronavirus. Springer-Verlag Berlin Heidelberg 2010, 1328, http://dx.doi.org/10.1007/978-3-642-03683-5.

7. Chen, S.; Lu, D.; Zhang, M.; Che, J; Yin, Z.; Zhang, S.; Zhang, W.; Bo, X.; Ding, Y.; Wang, S. Doubleantigen sandwich ELISA for detection of antibodies to SARS-associated coronavirus in human serum. Eur J Clin Microbiol Infect Dis 2005, 24, 549-553, https://doi.org/10.1007/s10096-005-1378-7.

8. $\quad$ Ren, Y.; Ding, H.; Wu, Q.; Chen, W.; Chen, D.; Bao, Z.; Yang, L.; Zhao, C.; Wang, J. [Detection of SARS-CoV RNA in stool samples of SARS patients by nest RT-PCR and its clinical value]. Zhongguo Yi Хие Ke Xие Yuan Xие Bao. 2003, 25, 368-371.

9. Gralinski, L.E.; Sheahan, T.P.; Morrison, T.E.; Menachery, V.D.; Jensen, K.; Leist, S.R.; Whitmore, A.; Heise, M.T.; Baric, S. Complement Activation Contributes to Severe Acute Respiratory Syndrome Coronavirus Pathogenesis. mBio. 2018, 9, 01753-18, https://doi.org/10.1128/mbio.01753-18.

10. Shin, G-C.; Chung, Y-S.; Kim, I-S.; Cho, H-W.; Kang, C. Preparation and characterization of a novel monoclonal antibody specific to severe acute respiratory syndrome-coronavirus nucleocapsid protein. Virus Res. 2006, 122, 109-118, https://doi.org/10.1016/j.virusres.2006.07.004.

11. Lei, J.; Hilgenfeld, R. RNA-virus proteases counteracting host innate immunity. FEBS Lett. 2017, 591, 3190-3210, https://doi.org/10.1002/1873-3468.12827.

12. Yang, H.; Yang, M.; Ding, Y.; Liu, Y.; Lou, Z.; Zhou, Z.; Sun, L.; Mo, L.; Ye, S.; Pang, H. \& al. The crystal structures of severe acute respiratory syndrome virus main protease and its complex with an inhibitor. Proc. Natl. Acad. Sci. U. S. A. 2003, 100, 13190-13195, https://doi.org/10.1073/pnas.1835675100.

13. Ishola, A.A.; Adedirin, O.; Joshi, T.; Chandra, S. QSAR modeling and pharmacoinformatics of SARS coronavirus 3C-like protease inhibitors. Comput. Biol. Med. 2021, 134, 104483, https://doi.org/10.1016/j.compbiomed.2021.104483.

14. Ercan, S.; Çınar, E. A molecular docking study of potential inhibitors and repurposed drugs against SARSCoV-2 main protease enzyme. J. Indian Chem. Soc. 2021, 98, 100041, https://doi.org/10.1016/j.jics.2021.100041.

15. El Aissouq, A.; Toufik, H.; Stitou, M.; Ouammou, A.; Lamchouri, F. In Silico Design of Novel TetraSubstituted Pyridinylimidazoles Derivatives as c-Jun N-Terminal Kinase-3 Inhibitors, Using 2D/3DQSAR Studies, Molecular Docking and ADMET Prediction. Int. J. Pept. Res. Ther. 2020, 26, 1335-1351, https://link.springer.com/article/10.1007/s10989-019-09939-8.

16. Ya'u Ibrahim, Z.; Uzairu, A.; Shallangwa, G.; Abechi, S. Molecular docking studies, drug-likeness and in-silico ADMET prediction of some novel $\beta$-Amino alcohol grafted 1,4,5-trisubstituted 1,2,3-triazoles derivatives as elevators of $\mathrm{p} 53$ protein levels. Sci. African 2020, 10, e00570, https://doi.org/10.1016/j.sciaf.2020.e00570.

17. Goudzal, A.; El Aissouq, A.; El Hamdani, H.; Ouammou, A. QSAR modeling, molecular docking studies and ADMET prediction on a series of phenylaminopyrimidine-(thio) urea derivatives as CK2 inhibitors. Mater. Today Proc. 2020, https://doi.org/10.1016/j.matpr.2020.08.044.

18. Kasmi, R.; Hadaji, E.; Chedadi, O.; El Aissouq, A.; Bouachrine, M.; Ouammou, A. 2D-QSAR and docking study of a series of coumarin derivatives as inhibitors of CDK (anticancer activity) with an application of the molecular docking method. Heliyon 2020, 6, e04514, 
https://doi.org/10.1016/j.heliyon.2020.e04514.

19. Chhatbar, D.M.; Chaube, U.J.; Vyas, V.K.; Bhatt, H.G. CoMFA, CoMSIA, Topomer CoMFA, HQSAR, molecular docking and molecular dynamics simulations study of triazine morpholino derivatives as mTOR inhibitors for the treatment of breast cancer. Comput. Biol. Chem. 2019, 80, 351-363, https://doi.org/10.1016/j.compbiolchem.2019.04.017.

20. Stitou, M.; Toufik, H.; Bouachrine, M.; Lamchouri, F. Quantitative structure-activity relationships analysis, homology modeling, docking and molecular dynamics studies of triterpenoid saponins as Kirsten rat sarcoma inhibitors. J. Biomol. Struct. Dyn. 2020, 39, 152-170, https://doi.org/10.1080/07391102.2019.1707122.

21. Konno, S.; Thanigaimalai, P.; Yamamoto, T.; Nakada, K.; Kakiuchi, R.; Takayama, K.; Yamazaki, Y.; Yakushiji, F.; Akaji, K.; Kiso, Y. \& al. Design and synthesis of new tripeptide-type SARS-CoV 3CL protease inhibitors containing an electrophilic arylketone moiety. Bioorg. Med. Chem. 2013, 21, 412-24, https://doi.org/10.1016/j.bmc.2012.11.017.

22. El Aissouq, A.; Toufik, H. \& al. QSAR study of isonicotinamides derivatives as Alzheimr's disease inhibitors using PLS-R and ANN methods. 2019 Int. Conf. Intell. Syst. Adv. Comput. Sci. (ISACS) 2019, 1-7, https://doi.org/10.1109/ISACS48493.2019.9068919.

23. Tong, J.; Zhan, P.; Wang, X.S.; Wu, Y. Quionolone carboxylic acid derivatives as HIV-1 integrase inhibitors: Docking-based HQSAR and topomer CoMFA analyses. J. Chemom. 2017, 31, e2934, https://doi.org/10.1002/cem.2934.

24. Clark, M.; Cramer, R.D.; Van Opdenbosch, N. Validation of the general purpose tripos 5.2 force field. $J$. Comput. Chem. 1989, 10, 982-1012, https://doi.org/10.1002/jcc.540100804.

25. Gasteiger, J.; Saller, H. Calculation of the Charge Distribution in Conjugated Systems by a Quantification of the Resonance Concept. Angew. Chemie Int. Ed. 1985, 24, 687-689, https://doi.org/10.1002/anie.198506871.

26. Cramer, R.D.; Patterson, D.E.; Bunce, J.D. Comparative Molecular Field Analysis (CoMFA). 1. Effect of Shape on Binding of Steroids to Carrier Proteins. J. Am. Chem. Soc. 1988, 110, 5959-5967, https://doi.org/10.1021/ja00226a005.

27. Pirhadi, S.; Shiri, F.; Ghasemi, J.B. Pharmacophore elucidation and 3D-QSAR analysis of a new class of highly potent inhibitors of acid ceramidase based on maximum common substructure and field fit alignment methods. J. Iran. Chem. Soc. 2014, 11, 1329-1336, http://dx.doi.org/10.1007/s13738-0130402-6.

28. Klebe, G.; Abraham, U.; Mietzner, T. Molecular Similarity Indices in a Comparative Analysis (CoMSIA) of Drug Molecules To Correlate and Predict Their Biological Activity. J. Med. Chem. 1994, 37, 41304146, https://doi.org/10.1021/jm00050a010.

29. Bush, B.L.; Nachbar, R.B. Sample-distance partial least squares: PLS optimized for many variables, with application to CoMFA. J. Comput. Aided. Mol. Des. 1993, 7, 587-619, https://doi.org/10.1007/bf00124364.

30. Wu, S.; Qi, W.; Su, R.; Li, T.; Lu, D.; He, Z. CoMFA and CoMSIA analysis of ACE-inhibitory, antimicrobial and bitter-tasting peptides. Eur. J. Med. Chem. 2014, 84, 100-106, https://doi.org/10.1016/j.ejmech.2014.07.015.

31. Oprea, T.I.; Waller, C.L.; Marshall, G.R. Three-Dimensional Quantitative Structure-Activity Relationship of Human Immunodeficiency Virus (I) Protease Inhibitors. 2. Predictive Power Using Limited Exploration of Alternate Binding Modes. J. Med. Chem. 1994, 37, 2206-2215, https://doi.org/10.1021/jm00040a013.

32. Begum, S.; Jaswanthi, P.; Venkata Lakshmi, B.; Bharathi, K. QSAR studies on indole-azole Analogues using DTC tools; imidazole ring is more favorable for aromatase inhibition. J. Indian Chem. Soc. 2021, 98, 100016, https://doi.org/10.1016/j.jics.2021.100016.

33. Yang, H.; Xie, W.; Xue, X.; Yang, K.; Ma, J.; Liang, W.; Zhao, Q.; Zhou, Z.; Pei, D.; Ziebuhr, J. \& al. Design of wide-spectrum inhibitors targeting coronavirus main proteases. PLoS Biol. 2005, 3, e324, https://doi.org/10.1371/journal.pbio.0030324.

34. Morris, G.M.; Huey, R.; Lindstrom, W.; Sanner, M.F.; Belew, R.K.; Goodsell, D.S.; Olson, A. AutoDock4 and AutoDockTools4 : Automated Docking with Selective Receptor Flexibility. J. Comput. Chem. 2009, 30, 2785-2791, https://doi.org/10.1002/jcc.21256.

35. Trott, O.; Olson, A.J. AutoDock Vina: Improving the Speed and Accuracy of Docking with a New Scoring Function, Efficient Optimization, and Multithreading. J. Comput. Chem. 2010, 31, 455-461, 
https://dx.doi.org/10.1002/jcc.21334.

36. DeLano, W.L. The PyMOL Graphics System. DeLano Scientific San Carlos, CA 2002.

37. Biovia Discovery Studio. Dassault Systèmes, https://www.3dsbiovia.com.

38. Briones, R.; Blau, C.; Kutzner, C.; de Groot, B.L.; Aponte-Santamaría, C. GROmaps: A GROMACSBased Toolset to Analyze Density Maps Derived from Molecular Dynamics Simulations. Biophys. J. 2019, 116, 4-11, https://doi.org/10.1016/j.bpj.2018.11.3126.

39. Vanommeslaeghe, K,; Hatcher, E.; Acharya, C.; Kundu, S.; Zhong, S.; Shim, J.; Darian, E.; Guvench, O.; Lopes, P.; Vorobyov, I.; Mackerell Jr., A.D. CHARMM General Force Field: A Force Field for DrugLike Molecules Compatible with the CHARMM All-Atom Additive Biological Force Fields. J. Comput. Chem 2010, 31, 671-690, https://doi.org/10.1002/jcc.21367.

40. Best, R.B.; Zhu, X.; Shim, J.; Lopes, P.E.M.; Mittal, J.; Feig, M.; Mackerell Jr., A.D. Optimization of the Additive CHARMM All-Atom Protein Force Field Targeting Improved Sampling of the Backbone $\phi, \psi$ and Side-Chain $\chi(1)$ and $\chi(2)$ Dihedral Angles. J. Chem. Theory Comput. 2012, 8, 3257-3273, https://doi.org/10.1021/ct300400x.

41. Jorgensen, W.L.; Chandrasekhar, J.; Madura, J.D.; Impey, R.W.; Klein, M.L. Comparison of simple potential functions for simulating liquid water. J. Chem. Phys. 1983, 79, 926-935, http://dx.doi.org/10.1063/1.445869.

42. Olasupo, S.B.; Uzairu, A.; Shallangwa, G.; Uba, S. QSAR modeling, molecular docking and ADMET/pharmacokinetic studies: a chemometrics approach to search for novel inhibitors of norepinephrine transporter as potent antipsychotic drugs. J. Iran. Chem. Soc. 2020, 17, 1953-1966, https://doi.org/10.1007/s13738-020-01902-5.

43. Larik, F.A.; Faisal, M.; Saeed, A.; Channar, P.A.; Korabecny, J.; Jabeen, F.; Mahar, I.A.; Kazi, M.A.; Abbas, Q.; Murtaza, G. \& al. Investigation on the effect of alkyl chain linked mono-thioureas as Jack bean urease inhibitors, SAR, pharmacokinetics ADMET parameters and molecular docking studies. Bioorg. Chem. 2019, 86, 473-481, https://doi.org/10.1016/j.bioorg.2019.02.011.

44. Domínguez-Villa, F.X.; Durán-Iturbide, N.A.; Ávila-Zárraga, J.G. Synthesis, molecular docking, and in silico ADME/Tox profiling studies of new 1-aryl-5-(3-azidopropyl)indol-4-ones: Potential inhibitors of SARS CoV-2 main protease. Bioorg. Chem. 2021, 106, 104497 , https://doi.org/10.1016/j.bioorg.2020.104497.

45. Nainwal, L.M.; Shaququzzaman, M.; Akhter, M.; Husain, A.; Parvez, S.; Khan, F.; Naematullah, M.; Alam, M.M. Synthesis, ADMET prediction and reverse screening study of 3,4,5-trimethoxy phenyl ring pendant sulfur-containing cyanopyrimidine derivatives as promising apoptosis inducing anticancer agents. Bioorg. Chem. 2020, 104, 104282, https://doi.org/10.1016/j.bioorg.2020.104282.

46. Sarma, P.; Sekhar, N.; Prajapat, M.; Avti, P.; Kaur, H.; Kumar, S.; Singh, S.; Kumar, H.; Prakash, A.; Dhibar, D.P.; Mehdi, B. In-silico homology assisted identification of inhibitor of RNA binding against 2019-nCoV N-protein (N terminal domain). J. Biomol. Struct. Dyn. 2020, 39, 2724-2732, https://doi.org/10.1080/07391102.2020.1753580.

47. Enayatkhani, M.; Hasaniazad, M.; Faezi, S.; Guklani, H.; Davoodian, P.; Ahmadi, N.; Einakian, M.A.; Karmostaji, A.; Ahmadi, K. Reverse vaccinology approach to design a novel multi-epitope vaccine candidate against COVID-19: an in silico study. J. Biomol. Struct. Dyn. 2020, 39, 2857-2872, https://doi.org/10.1080/07391102.2020.1756411.

48. Elmezayen, A.D.; Al-Obaidi, A.; Şahin, A.T.; Yelekçi, K. Drug repurposing for coronavirus (COVID19): in silico screening of known drugs against coronavirus 3CL hydrolase and protease enzymes. $J$. Biomol. Struct. Dyn. 2020, 39, 2980-2992, https://doi.org/10.1080/07391102.2020.1758791. 